kommen durchsichtig und anscheinend monoklin sind. So dargestellt, enthält es aber 2wei Moleküle Krystallwasser:

0.3301 g Sbst.: $0.0932 \mathrm{~g} \mathrm{Pt}$.

$\left(\mathrm{C}_{6} \mathrm{H}_{13} \mathrm{ON}\right)_{2} \cdot \mathrm{H}_{2} \mathrm{PtCl}_{6}+2 \mathrm{H}_{2}$ O. Ber. Pt 28.6. Gef. Pt 28.2.

Das Salz wurde an der Luft auf Thon getrocknet. Eine andere Probe wurde zerrieben und etwa zwei Wochen über Chlorcalcium aufbewahrt. Es erwies sich dann als wasserfrei:

$0.2343 \mathrm{~g}$ Sbst.: $0.0716 \mathrm{~g} \mathrm{Pt}$.

$\left(\mathrm{C}_{6} \mathrm{H}_{15} \mathrm{ON}\right)_{2} . \mathrm{H}_{2} \mathrm{PtCl}_{6}$ Ber. Pt 30.20. Gef. Pt 30.56.

168. Arthur Lachman: Ueber das Bewad'sohe Triäthylaminoxyd.

[Mittheilung aus dem chemisohen Laboratorium der University of Oregon.] (Eingeg. am 30. Marz; mitgeth. in der Sitzung von Brn. R. Wolffenstein.)

Aus den in der vorangehenden Mittheilung erwähnten Gründen ist es mir nicht möglich, die ursprünglich geplante ausführliche Untersuchung über das Bewad'sche Triäthylaminoxyd fortzusetzen; um aber den mit den Arninoxyden beschäftigten Forschern einige Mübe und Zeit zu ersparen, wird eine Mittheilung meiner Darstellungsmethode und sonstigen Beobachtungen vielleicht willkommen sein.

Wie in der vorangehenden Abhandlung dargethan ist, haben Dunstan und Goulding höchst wahrscheinlich ein Isomeres dieser Bewad'schen Verbindung entdeckt. Bewad selbst hat seine Verbindung analysirt und durch Reduction zn Triäthylamin die Bindungsart der drei Aethylgruppen nachgewiesen. Dasselbe haben Dunstan und Goulding mit den von ihnen entdeckten Körpern gethan, sodass es nach den bis jetzt mitgetheilten Erfahrungen $z$ wei Substanzen genau derselben Constitution

$$
\begin{aligned}
& \mathrm{C}_{2} \mathrm{H}_{5} \\
& \mathrm{C}_{2} \mathrm{H}_{5}-\mathrm{N}: \mathrm{O} \\
& \mathrm{C}_{2} \mathrm{H}_{3}
\end{aligned}
$$

giebt. In der That besitzen diese beiden Verbindungen grundverschiedene Eigenschaften. Allerdings bleibt die thatsăchliche Identität der Zusammensetzung noch schärfer zu beweisen, obwohl diese Constatirung dritterseits nur zur Verstärkung der Gewissheit beitragen kann. Die năchstliegende Annahme, die Dunstan-Goulding'sche Verbindung sei ein Aminoxydhydrat:

$$
\left(\mathrm{C}_{\mathbf{2}} \mathrm{H}_{\mathbf{s}}\right)_{\mathbf{3}} \mathrm{N}<\mathrm{OH} \text {, }
$$

während der ălteren Verbindung die wirkliche Aminoxydformel zukommt, wird dadurch widerlegt, dass beide Körper ans Halogen- 
wasserstoffsalzen, also aus Substanzen genan gleicher Constitution, durch Basen in Freibeit gesetzt werden. Die Aufklärung der Isomerie steht demnach noch aus.

\section{Darstellung des Triathylaminoxyds.}

Wie schon vor einiger Zeit mitgetheilt wurde 1), erhält man bei der Darstellung des Triäthylaminoxyds eine weit bessere Ausbente, auf Nitroäthan berechnet, wenn man die von Bewad ') empfoblene Menge Zinkäthyl (nämlich ein Molekül) verdoppelt. $Z_{w a r}$ giebt Bewad an, dass unter solehen Umstănden kein Triäthylaminoxyd, sondern ein neutraler Körper entsteht; darin befindet sich aber Bewad im Irrtbum, denn ich erhielt immer vorzügliche Ausbeuten an Aminoxyd und fand keine Spur des zweiten Körpers. Da nun ferner das Zinkäthyl, nach der von mir angegebenen Methode ${ }^{\S}$ ), ein bei Weitem leichter darzustellender Körper als das Nitroăthan ist, so empfiehlt es sich auch aus ökonomischen Rũcksichten, das Nitroüthan auf Kosten des Zinkäthyls auszunutzen. Zu erwähnen bleibt aber, dass unter solchen Umständen das von $B$ ew ad bescbriebene Additionsproduct nicht zur Ausscheidung gelangt; zu dessen Darstellang ist also die ursprüngliche Anordnung beizubebalten.

In einen mit Kohlensäure gefülltem Halbliterkolben giebt man $150 \mathrm{~g}$ Zinkäthyl und etwa $200 \mathrm{ccm}$ trocknen Aether ${ }^{4}$ ). Nach Zugabe der enteprechenden Menge Nitroäthan ( $46 \mathrm{~g}$ ) verschliesst man den Kolben mit Korkstopfen und Steigrohr, welches Letztere zu einer nicht zu engen Capillare auszuziehen ist, da es sich leicht verstopft. Vorsichtshalber lässt man über Nacht in Wasser stehen (einmal ist mir ein Kolben in die Luft gegangen), und stellt dann den Kolben an einen dunklen, küblen Ort bin. Der Verlauf der Reaction lăsst sich durch eine gleichzeitig erfolgende Farbenveränderung leicht verfolgen. Beim ersten Vermischen tritt sofort eine ziemlich intensive Gelbfärbung anf, welche allmählich verblasst. Nach etwa 14-16 Tagen ist die Flüssigkeit fast farblos und dann zur Weiterverarbeitung bereit. Es empfiehlt sich, etwa einmal tãglich den Kolben umzuschwenken, um einen nicht unerheblichen, amorphen, weissen Boden-

1) Amer. Chem. Journ. 21, 435.

2) Diese Berichte 21, Ref. 479; 22, Ref. 250.

3) Eine kurze Beschreibung dieser Methode befindet sich im Amer. Chem. Journ. 19, 410. Eine ausführliche Mittheilung soll demnăchst erscheinen.

4) Nach Squibb (das Citat ist mir entfallen) kann man Aether mittels Chlorcalcium vollstăndig entwăssern. Ich habe diese Methode seit Jahren gebraucht and vorzüglich gefunden. Wendet man Aether vom Volumgewicht $0.720 \mathrm{~g}$ an, so ist die Entwhsserung in einer Woche vollendet, und zum Grebrauch uar abzufiltriren. Man kann sich auf diese Weise leicht grosse Mengen Aethers in trocknem Zustande vorrathig halten. 
satz zu vertheilen. Zur Isolirung des Triäthylaminoxyds giebt man mun in den Kolben etwa $300 \mathrm{ccm}$ Aetber und giesst das Gemisch langsam, unter tïchtigem Unrühreu und Abkühlen, in folgende Lösung: A mmoniumsulfat $250 \mathrm{~g}$, Ammoniak fü̈ssigk eit $100 \mathrm{ccm}$, Wasser $500 \mathrm{ccm}$. In dieser Lösung ist das sofort ausgeschiedene Zinkhydroxyd leicht löslich; diese Methode ist deshalb bei allen Zinkätbylsyntbesen sebr zu empfehlen ${ }^{1}$ ). Zum Schluss schüttelt man in Scheidetrichter tüchtig durch und äthert den Rückstand noch einmal aus. Die rereinigten Auszüge werden mehrere Male mit Salzsäure extrahirt, um sicher alle Base dem $\Lambda$ ether zu entziehen; aus den Aether kamn mas durrb Verdunsten etwa $7 \mathrm{~g}$ Nitroäthan zurückgewinnen. Die salzsaure Lösung wird dann 4-5 Stunden auf dem Wasserbade eingedampft. Diese letztere Bebandlung ist bei Leibe nicht zu unterlassen; sonstigen Falls geht in die gewünschte Base ein Körper über, welcher bei der Destillation den totalen Zerfall des Aminoxyds (wie es scheint, katalytisch) verursacht. Ich habe diese Erfahrung auf Kosten von $40 \mathrm{~g}$ der werthvollen Base gemacht. Durch die linge Erwärmung mit überschüssiger Säure wird dieser Körper zerstört.

Das dunkelgefürbte Hydrochlorat bringt man in überschüssige, starke Natronlauge und äthert aus, sobald die Flüssigkeit sich genügend abgekühlt hat. Die Hauptmenge des Aminoxyds schwinnt direct auf der Lauge und die geringe, noch in Lösung befindliche Menge wird durch zweimaliges Ausätbern leicht gewonnen. Man trocknet über Pottasche oder Aetzkali, destillirt den Aether vorsichtig ab (die Base ist mit Aether flüchtig) und fractionirt den Rückstand zwei r,is drei Mal. Die Ausbeute an reinem Triätbylaminoxyd beträgt etwa $30 \mathrm{~g}$, statt $72 \mathrm{~g}$ berechnet ( $42 \mathrm{pCt}$. der Theorie). Berücksichtigt man das zurückgewonnene Nitroäthan, so erhöht sich die Ausbeute auf $48 \mathrm{pCt}$.

\section{Eigenschaften des Triäthylaminoxyds.}

Leider habe ich im Anfang andere Ziele verfolgt, als das Triätbylaminoxyd selbst näber zu beschreiben, und da ich später die Hauptmenge meines kostbaren Materials verloren habe, bietet sich jetzt keine Gelegenheit mebr, das Versäumte nachzuholen. Daher kann ich nur Bruchstücke beitragen; das Wenige wird aber vielleicht Anderen nützlich sein.

1) Der Hauptrortheil dieser Methode liegt in der bedeutend geringeren Wärmenienge, welche bei der Zerset\%ung des Zinkäthyls frei wird. Sie ist in allen Fällen zu getrauchen, in welchen das gesuchte Product von Ammoniak nicht angegriffen wird und in Wasser nicht übermässig löslich ist. Letzterer Umstand verbietet ihre Anwendung beim Diäthylhydroxylamin. 
Analyse. Das zu diesem $Z$ wecke gebrauchte Material war frisch destillirt, farblos und rom richtigen Siedepunkt. Immerhin lieferte die Stickstoffuestimmung nach Dumas zu hohe Zahlen.

$0.1365 \mathrm{~g}$ Sbst.: $15.6 \mathrm{ccm} \mathrm{N}\left(15.5^{\circ}, 751 \mathrm{~mm}\right) .-0.1092 \mathrm{~g}$ Sbst.: $13.0 \mathrm{ccm} \mathrm{N}$ $\left(18^{0}, 746 \mathrm{~mm}\right)$.

$$
\mathrm{C}_{6} \mathrm{H}_{15} \mathrm{ON} \text {. Ber. } \mathrm{N} \text { 12.00. Gef. N 13.21, 13.52. }
$$

Es sei erwähnt, dass die Alwesenheit von Stickoxyden besonders festgestellt wurde. Das Triäthylaminoxyd scheint sich demnach gerade wie das Diäthylhydroxylamin zu verhalten, und wobl wabrscheinlich aus demselben Grunde.

Während aber das Diäthylhydroxylamin nach K jeldahl-Krüger die berechnete Menge Ammoniak lieferte, treten bei dem Aminoxyd sofort Stickoxyde auf, wenn die Chromsäure einzuwirken beginnt. Diese Oxyde sind durch Geruch, Aussehen und Jodkaliumstärkepapier identificirt worden. Daher wurde nach dieser Methode viel zu wenig Stickstoff gefunden.

\section{Ber. N 12.00 Gef. N 7.00.}

Es ist die Thatsache ron einiger Wicbtigkeit in Bezug auf die Haftenergie des Sauerstoffs.

Salzsaures Salz. Versucht man durch Verdunsten berechneter Mengen Base und Salzsäure das salzsaure Salz zu erhalten, so verbleibt selbst nach peinlichster Vorsicht und mehrwöchentlichem Verweilen im Exsiccator nur ein gummiartiger Rückstand. Dampft man dagegen, wie in der Bewad'schen Vorscbrift, eine stark saure Lösung zur Syrupdicke ein und lässt dann im Exsiccator stehen, so erhält man zwar grosse, prismatische Krystalle, die jedoch ungemein zerfliesslich sind und so viel der sauren Mutterlauge einschliessen, dass sie zur Analyse nngeeignet sind.

Verbalten in der Wärme. Das reine Aminoxyd, wie schon früher mitgetheilt, ist unzersetzt flüchtig und besitzt bei $753 \mathrm{~mm}$ den corrigirten Sdp. 15?-158 . Bei der oben erwähnten katalytischen Zersetzung wurde jedoch benbachtet, dass eine Fraction vom ungefäbr richtigen Siedepunkt bei wiederholtem Destilliren schon unter $100^{\circ}$ basische Dämpfe entwickelte, die sich wie Triäthylamin verbielten, während andererseits ein beträchtlicher Rückstand nunmebr verblieb, welcher nicht mehr ohne starke Zersetzung flüchtig war. Entwickelung von Sauerstoff konnte nicht beobachtet werden. Es scheint also, dass unter diesen Umstinden das Triăthylaminoxyd $\mathbf{z w a r}$ nach folgender Gleichung in Triäthylamin und Sauerstoff zerfiel:

$$
\left(\mathrm{C}_{2} \mathrm{H}_{3}\right)_{3} \mathrm{NO}=\left(\mathrm{C}_{2} \mathrm{H}_{3}\right)_{3} \mathrm{~N}+\mathrm{O},
$$

dass aber der Sauerstoff unter Bildung hochsiedender Oxydationsproducte verbraucht wurde.

Berichte d. D. chem. Gesellsehaft. Jahry. XXXIII. 
Addition von Jodmethyl. Diese Reaction entwickelt binnen Kurzem soviel Wärme, dass das Gemisch zu sieden anfängt. Bringt man die Körper unter Kühlung zusammen und bewahrt einige Tage im Dunkeln auf, so wird viel Jod frei, und es bildet sich jodwasserstoffsaures Triäthylamin. Es entspricht dies den beiden Reactionen (unter Annabme vorangehender Addition) ${ }^{1}$ ):

I. $\left(\mathrm{C}_{2} \mathrm{H}_{5}\right)_{3} \mathrm{~N}<\mathrm{J} \cdot \mathrm{CH}_{3}=\left(\mathrm{C}_{2} \mathrm{H}_{3}\right)_{3} \mathrm{~N} \cdot \mathrm{HJ}+\mathrm{CH}_{2} \mathrm{O}$.

II. $\left(\mathrm{C}_{8} \mathrm{H}_{3}\right)_{3} \mathrm{~N}<\mathrm{J} \cdot \mathrm{CH}_{3}+2 \mathrm{CH}_{3} \mathrm{~J}$

$$
=\left(\mathrm{C}_{2} \mathrm{H}_{5}\right)_{3}\left(\mathrm{CH}_{3}\right) \mathrm{N} . \mathrm{J}+\mathrm{J}_{2}+\left(\mathrm{C}_{2} \mathrm{H}_{6} \mathrm{O}\right) ?
$$

Die erhaltene Menge Triätbylamin entsprach etwa $17 \mathrm{pCt}$. der nach Gleichung I berechneten; das freie Jod entsprach $32 \mathrm{pCt}$. der zweiten Gleichung. Leider wurde ich durch Krankheit an der Weiterführung dieser Reaction rerhindert.

Condensationsreactionen. Triäthylaminoxyd wurde $7 \mathrm{Stdn}$. auf $170^{\circ}$ mit Anilin und Phenylhydrazin erhitzt, obne dass sich irgend eine Reaction nachweisen liess. Es weist dies auf eine ziemlich starke Haftenergie des Sauerstoffs bin.

Oxydations- und Reductions-Erscheinungen. Folgende Reactionen wurden mit einer Lösung des salzsauren Salzes ausgeführt.

Fehling'sche Lösung: sofort in der Kälte reducirt.

Ammoniakalisches Silbernitrat: beim Erwärmen Silberspiegel.

Jodwasserstoff wird nach kurzem Kochen nicht reducirt.

Bichromat, Permanganat: sofort reducirt.

Zinnchlorür: in saurer Lösung ohne Einwirkung.

Zinnchlorür: in alkalischer Lösung schwach reducirt.

Bromwasser: sofort entfärbt.

Eisenchlorid: ohne Einwirkung.

Quecksilberchlorid: ohne Einwirkung.

Natriumnitrit: giebt sofort Stickoxyd.

Aus dieser Zusammenstellung ersieht man, dass in der That das Bewad'sche Aminoxyd den Hydroxylaminen, speciell dem Diäthylhydroxylamin, sehr nahe verwandt sein muss. Dadurch wird die Isomerie mit dem Dunstan-Goulding'schen Triäthyloxamin nur räthselhafter. Zunächst muss aber durch einwandsfreie Analysen beider Basen die Isomerie wirklich sicher gestellt werden.

1) Man vergleiche Bamberger und Tschirner, diese Berichte $32,18 \$ 2$, wo unter ähnlichen Umständen bei dem Dimethylanilinoxyd Formaldehyd direct nachgewiesen wurde. 